\title{
Corneal squamous cell carcinoma in a dog
}

\author{
João Antonio Tadeu Pigatto', Paula Stieven Hünning', Fabiana Quartiero Pereira', Ana Carolina \\ Rodarte da Veiga de Almeida', Cristiano Gomes', Luciane Albuquerque' \& David Driemeier ${ }^{2}$
}

\begin{abstract}
Background: Squamous cell carcinoma (SCC) is a neoplastic disease of the squamous epithelial cells that has been rarely described in the literature. This neoplasm affects the eyelid, conjunctiva and third eyelid, as well as the cornea. Corneal SCC is a neoplastic lesion characterized by a pink, typically irregular mass protruding from the epithelial surface of the cornea. Canine corneal SCC has been associated with chronic keratoconjunctivitis sicca, or keratitis secondary to exophthalmia and is common in humans, horses and cows. The treatment is surgical with excision of the lesion of the ocular surface. This paper reported a study of a dog with corneal SCC, which was successfully treated with a superficial lamellar keratectomy excision combined with cryosurgery.

Case: An 8-year-old male English bulldog was presented for the evaluation of a red mass on its left eye that had progressively grown over a 1-year period. The mass was approximately $6 \mathrm{~mm}$ in diameter and it was elevated from 3 to $4 \mathrm{~mm}$ above the corneal surface with no expansion onto bulbar conjunctiva. At physical examination it was normal except for the ocular disease. A complete blood cell count and serum chemical profiles were unremarkable. The radiographic evaluation did not demonstrate any evidence of metastasis. The mass was excised by a superficial lamellar keratectomy and the surgical bed was frozen with nitrous oxide. The surgical procedures consisted in excising a rim of grossly normal conjunctiva with $2 \mathrm{~mm}$ along the mass that was sent for histopathological examination. The postoperative measures included the administration of topical tobramycin at $0.3 \%$, four times a day, and systemic carprofen $4 \mathrm{mg} / \mathrm{kg}$, daily, for 5 days. A reexamination was performed after $14,21,30,42,70$ and 180 days postoperatively. When the cornea was healed, topical dexamethasone at $1 \%$ was prescribed, twice a day for 2 weeks to control an excessive vascularization at the limbus. In 70 days, the postoperative result was very satisfactory and there was no evidence of ocular inflammation. Two years after the surgical procedure the dog had not developed metastasis or recorrence of the ocular neoplasm.

Discussion: Ocular or adnexal SCC in animals and humans is suspected to be a result of the chronic effect of ultraviolet light on the epithelium. Also, in dogs the SCC has been associated with a chronic source of irritation. The dog observed in our study was brachycephalic, with natural exophthalmic eyes and oversized palpebral fissures that may have caused excessive and chronic corneal exposure to solar radiation. The treatment of choice was excision of the lesion with a wide surgical margin, because it is the standard treatment for SCC of the cornea and cryotherapy has been a common modality of treatment. A histological examination revealed islands and nests of pleomorphic epithelial cells and keratin pearls and the diagnosis of the corneal SCC was made. No evidence of metastasis or recurrence has been found since the surgical procedure. Although, recurrence rates following the excision of this neoplasm of the ocular surface ranged an average of $30 \%$. The treatment of the neoplasm was successful in both procedures, once no visible evidence of regrowth presented two years after the keratectomy. Despite being a rare entity, squamous cell carcinoma of cornea should be considered as differential diagnosis in dogs with any corneal mass.
\end{abstract}

Keywords: ocular neoplasm, canine, lamellar keratectomy, cryotherapy.

Descritores: neoplasia ocular, canino, ceratectomia lamellar, crioterapia. 


\section{INTRODUCTION}

Squamous cell carcinoma (SCC) is a neoplastic disease of squamous epithelial cells. In the ocular region, the eyelid, conjunctiva and third eyelid may be affected $[2,3,8]$. However, corneal SCC has been very rarely described $[2,8]$. Corneal SCC is a neoplastic lesion characterized by a pink, typically irregular mass protruding from the epithelial surface of the cornea $[3,8]$. The tumor is often found in areas of chronic inflammation or in those that have been under sun exposure. Canine corneal SCC has been associated with chronic keratoconjunctivitis sicca, or keratitis secondary to exophthalmia [1-4,8]. The excision of the lesion has been the most accepted method of treatment for SCC of the ocular surface. SCC of the cornea is common in horses and cows $[1,4,9]$. This paper has reported a study of a dog with corneal SCC, which was successfully treated with a superficial lamellar keratectomy excision combined with cryosurgery.

\section{CASE}

An 8-year-old male English bulldog was presented with a history of having some mass in its left eye that had progressively grown over a 1-year period. There was no previous treatment prior to our examination. The dog was well nourished and presented good clinical condition. A pupillary light reflex and menace responses were intact bilaterally. The ophthalmologic examination revealed a spherical red mass in the ventrolateral aspect of the cornea (Figure 1). The mass was approximately $6 \mathrm{~mm}$ in diameter and it was elevated from 3 to $4 \mathrm{~mm}$ above the corneal surface with no expansion onto bulbar conjunctiva. Large blood vessels extended from the limbus into the base of the mass and the limbus was free from it. The indirect ophthalmoscopic examination of the right eye was normal and it could not be adequately visualized in the left eye because of the corneal mass. At physical examination it was normal except for the ocular disease. Regional lymph nodes were not enlarged and the thoracic auscultation and the abdominal palpation revealed no abnormality. A complete blood cell count and serum chemical profiles were unremarkable. Thoracic, abdominal, and skull radiography did not demonstrate any evidence of metastasis.
The dog was anesthetized and the eye was routinely prepared for surgery. The lesion was delineated using a set depth knife. With the help of an operating microscope, the mass was excised with a clear margin. A rim of grossly normal conjunctiva with $2 \mathrm{~mm}$ was excised along the mass to ensure a complete excision, and the mass was sent for histopathological examination. By using nitrous oxide, the keratectomy site was treated with double freeze-thaw cryotherapy. The surgical wound was left apart, healing, for a second intention. In addition, the third eyelid flap was performed to protect the cornea. After the surgery, the eye was very comfortable presenting a minimal discharge. The medical treatment was administered as described. The dog was discharged 1 day after the procedure with topical tobramycin at $0.3 \%$ eye drops, QID and systemic carprofen $4 \mathrm{mg} /$ $\mathrm{kg}$, daily, for 5 days. A reexamination was performed after 14, 21, 30, 42, 70 and 180 days postoperatively, revealing an uncomplicated healing of the lesion. After 2 weeks, the third-eyelid flap was removed, and the cornea was healed, therefore, the topical antibiotic therapy was discontinued. After the second reexamination, the cornea was fluorescein-negative, and topical dexamethasone at $1 \%$ was prescribed (BID for 2 weeks, then SID for 2 weeks) to control an excessive vascularization at the limbus.

A histological examination revealed islands and nests of pleomorphic epithelial cells and keratin pearls (Figure 2). In 70 days, the postoperative result was very satisfactory and there was no evidence of

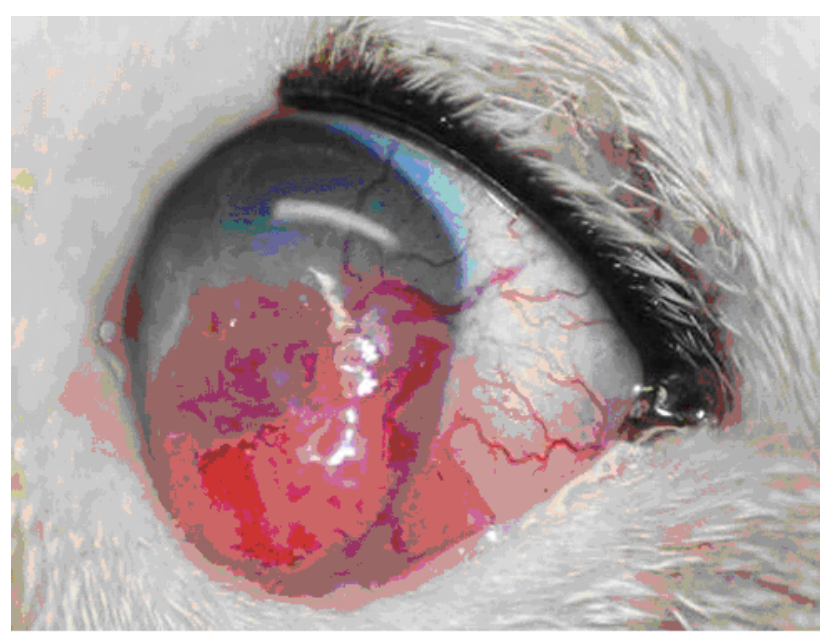

Figure 1. Gross appearance of the corneal squamous cell carcinoma in the left eye. 
ocular inflammation (Figure 3). The eye was visual 24 months after the surgery. Two years after the surgical procedure the dog demonstrated to be clinically normal, and thoracic and abdominal radiography did not show any evidence of metastasis.

\section{DISCUSSION}

The SCC of the cornea is common in humans, horses and cows, but it has been rarely reported in dogs in the literature $[2,4,8,9]$. In dogs, ocular SCC often involves the eyelid and conjunctiva, with a corneal involvement that is usually secondary to a spread limbal mass [3]. Ocular or adnexal SCC in animals and humans is suspected to be a result of the chronic effect of ultraviolet light on the epithelium. In dogs, SCC has been associated with a chronic source of irritation, keratoconjunctivitis sicca, keratitis

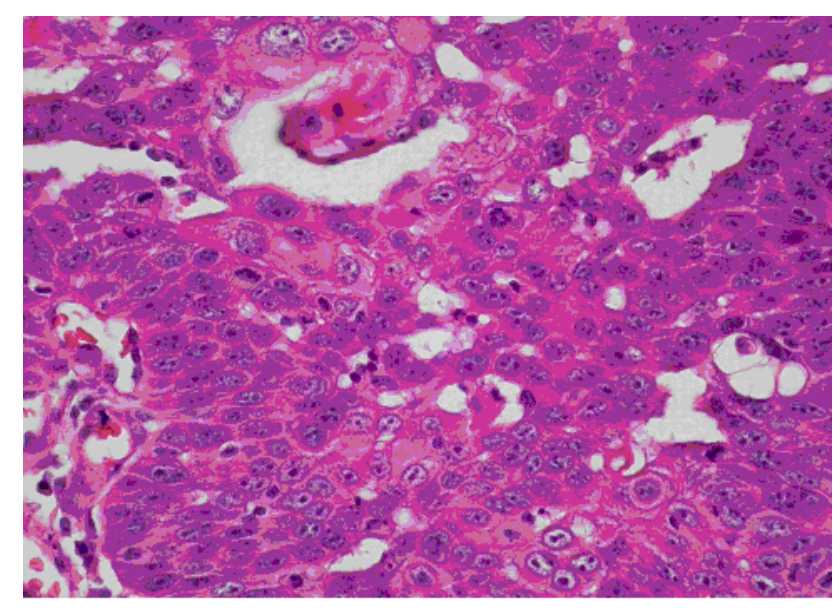

Figure 2. Light microphotograph of corneal squamous cell carcinoma showing intracellular and extracellular keratinization forming keratin pearls.

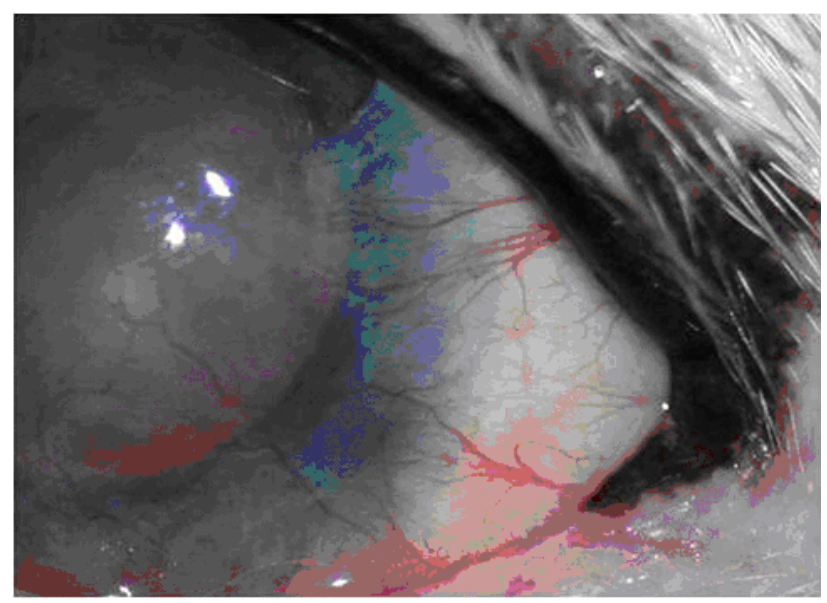

Figure 3. Macroscopic image of left eye 70 days after surgical procedure.. secondary to exophthalmia or trauma $[2-4,8]$. The dog observed in our study was brachycephalic, with natural exophthalmic eyes and oversized palpebral fissures that may have caused excessive and chronic corneal exposure to solar radiation. In the study reported, no source of irritation was found.

Corneal SCC is characterized by a pink, typically irregular mass protruding from the epithelial surface of the cornea $[3,8,11]$. In this study, clinical features and the biological behavior were similar to other previous reports. Corneal SCC metastasizes to regional lymph nodes, salivary glands and thorax, and may occur in horses up to $15 \%[6,12]$. No good estimate of metastasis rate has been reported for dogs. In the present study, thoracic and abdominal radiography demonstrated no evidence of metastasis. SCC may be suspicious based on the gross appearance of a lesion as well as its location, but a definitive diagnosis would require a microscopic examination of the affected tissue [13]. In the present study, the diagnosis was based on history and clinical signs and it was confirmed in the histopathology of the mass after surgical excision. Differential diagnoses for the corneal mass include papilloma, hemangioma, hemangiosarcoma, limbal melanoma, chronic superficial keratitis, episcleritis, corneal dermoid, epithelial inclusion cysts, and infectious keratitis $[2,3,7,8,10]$.

The histopathological features of SCC are characterized by invasive malignant squamous cells that violate the basement membrane and grow in sheets or cords into the stromal tissue [3]. In this study, a micro invasive well differentiated SCC forming a keratin pearl was perceived. The histological appearance was similar to that previously described. Several options of available treatments for SCC of the cornea have been a wide surgical excision alone or in combination with radiation therapy, cryotherapy, local hyperthermia or topical chemotherapy $[3,7,9$, 11,13]. The excision of the lesion with a wide surgical margin was the standard treatment for SCC of the cornea [7,12]. The major risk factor for recurrence has been identified as an inadequate excision margin. Recurrence rates following the excision of the ocular surface squamous neoplasia ranged from $15 \%$ to $52 \%$, with an average of $30 \%$ [12]. Cryotherapy has been also applied as a common modality of treatment $[7,9,11]$. The tumor was removed through lamellar keratectomy in the dog of the present report, and the surgical bed was subjected to double freeze-thaw 
cryotheraphy. An alternative treatment such as a topically applied radio frequency hyperthermia or an external irradiation was excluded because of the lack of appropriate equipment. Complications following cryotherapy such as iritis, decreased the intraocular pressure, and a thermic inflammatory edema and a scarred corneal have been related [12]. In this study, however, such complications were not observed. Thus, keratectomy was performed by a sharp incision with $2 \mathrm{~mm}$ beyond the visible corneal limits of the tumor, followed by the cryotherapy of the surgical bed $[12,13]$. Keratectomy started in the clear cornea and extended towards the limbus; the bulbar conjunctiva and the limbus were excised through sharp dissection. The keratectomy as well as the clear cornea provided a visual assurance of a tumor-free excision. The treatment of the neoplasm was successful in both procedures because of the tumor no regrowth, and it was not clinically discernable 2 years after the treatment. Further prospective studies are warranted in order to validate this option of treatment for this type of population. Corneal SCC should be included as a differential diagnosis of corneal diseases in dogs.

\section{REFERENCES}

1 Bailey C.M., Hanks D.R. \& Hanks M.A. 1990. Circumocular pigmentation and incidence of ocular squamous cell tumours in Bos taurus and Bos indicus $\times$ Bos taurus cattle. Journal of the American Veterinary Medical Association. 196(10): 1605-1608.

2 Bernays M.E., Flemming D. \& Peiffer R.L. 1999. Primary corneal papilloma and squamous cell carcinoma associated with pigmentary keratitis in four dogs. Journal of the American Veterinary Medical Association. 214(2): 215-217.

3 Busse C., Sansom J., Dubielzig R.R. \& Hayes A. 2008. Corneal squamous cell carcinoma in a Border Collie. Veterinary Ophthalmology. 11(1): 55-58.

4 Dugan S.J., Curtis C.R., Roberts S.M. \& Severin G.A. 1991. Epidemiologic study of ocular/adnexal squamous cell carcinoma in horses. Journal of the American Veterinary Medical Association. 198(2): 251-256.

5 Hargis A.M., Lee A.C. \& Thomassen R.W. 1978. Tumor and tumor-like lesions of perilimbal conjunctiva in laboratory dogs. Journal of the American Veterinary Medical Association. 173(9): 1185-1190.

6 Hendrix D.V.H. 2005. Equine ocular squamous cell carcinoma. Clinical Techniques in Equine Practice. 4(1): 87-94.

7 Latimer K.S., Kaswan R.L. \& Sundberg J.P. 1987. Corneal squamous cell carcinoma in a dog. Journal of the American Veterinary Medicine Association. 190(11): 1430-1432.

8 Montiani-Fereira F., Kiupel M., Muzolon P. \& Truppel J. 2008. Corneal squamous cell carcinoma in a dog: a case report. Veterinary Ophthalmology. 11(4): 269-272.

9 Ollivier F.J., Kallberg M.E., Plummer C.E., Barrie K.P., O’Reilly S., Taylor D.P., Gelatt K. \& Brooks D.E. 2006. Amniotic membrane transplantation for corneal surface reconstruction after excision of corneolimbal squamous cell carcinomas in nine horses. Veterinary Ophthalmology. 9(6): 404-413.

10 Paulsen M.E., Lavach J.D., Snyder S.P., Severin G.A. \& Eichenbaum J.D. 1987. Nodular granulomatous episclerokeratitis in dogs: 19 cases (1973-1985). Journal of the American Veterinary Medical Association. 190(12): 1581-1587.

11 Plummer C.E., Smith S., Andrew S.E., Lassaline M.E., Gelatt K.N., Brooks D.E., Kalberg M.E., Ollivier F.J. 2007. Combined keratectomy strontium-90 irradiation and permanent bulbar conjunctival grafts of corneolimbal squamous cell carcinomas in horses: 38 horses. Veterinary Ophthalmology. 10(1): 37-42.

12 Shin H.J., Sohn J.h., Goo S., Park J.Y., Choi C.H., Kim E.K., Cho S.H., Yoo N.C. \& Roth J.K. 2001. Squamous cell carcinoma of the cornea. Yonsei Medical Journal. 42(5): 576-579.

13 Ward D.A., Kenneth S.L. \& Askren R.M. Squamous cell carcinoma of the corneoscleral limbus in a dog. Journal of the American Veterinary Medical Association. 200(10): 1503-1506. 